\title{
LA VIOLENCIA ESCOLAR DESDE LAS REPRESENTACIONES SOCIALES DE ESTUDIANTES DE LA INSTITUCIÓN EDUCATIVA COLEGIO NUESTRA SEÑORA DE BELÉN*
}

\author{
SCHOOL VIOLENCE FROM THE SOCIAL REPRESENTATIONS OF STUDENTS \\ OF THE NUESTRA SEÑORA DE BELEN EDUCATIONAL INSTITUTION
}

\author{
Jesús ERnESTO URBINA-CÁrdenAS** \\ LuCyraima Beltrán-Castellanos***
}

\begin{abstract}
Resumen
Objetivo. Comprender las representaciones sociales sobre violencia escolar en estudiantes de la Institución Educativa Nuestra Señora de Belén de la ciudad de Cúcuta. Metodología. Estudio cualitativo desde el enfoque procesual de la teoría de las representaciones sociales. Resultados. Los estudiantes con conductas agresivas consideran que ninguna estrategia de prevención logrará un impacto favorable frente al fenómeno violento, teniendo en cuenta que representa un mecanismo de supervivencia donde estas conductas son aceptadas; mientras que los estudiantes no agresivos sugieren que las estrategias de prevención tendrían efectos positivos si además del castigo, permitieran la participación activa y el acompañamiento de las familias. Conclusión. Es necesario replantear el papel de la escuela como escenario para aprender a resolver los conflictos de manera pacífica, de modo que la naturalización de la violencia, el silencio de los afectados y la no autorregulación de las emociones no constituyan prácticas cotidianas socialmente aceptada.
\end{abstract}

Palabras clave: representaciones sociales, violencia, violencia escolar.

\begin{abstract}
Objective. To understand the social representations about school violence in students of the Nuestra Señora de Belén Educational Institution in the city of Cúcuta, Department of Santander. Methodology. Qualitative study from the processual approach of the theory of social representations. Results. The students with aggressive behaviors consider that no prevention strategy will achieve a favorable impact in the face of the violent phenomenon, considering that it represents a survival mechanism where these behaviors are accepted. The non-aggressive students suggest that prevention strategies would have positive effects if, in addition to punishment, they allowed the active participation and accompaniment of families. Conclusion. It is necessary to rethink the role of the school as a setting for learning to solve conflicts peacefully, so that the naturalization of violence, the silence of those affected and the non-self-regulation of emotions do not constitute a socially accepted daily practice.
\end{abstract}

Key words: social representations, violence, school violence.

\footnotetext{
*El artículo se origina en la investigación titulada "El dilema de la guerra o la paz: representaciones de niños, niñas y jóvenes sobre la guerra y la paz en Norte de Santander”, proyecto financiado por el Fondo de Investigaciones de la Universidad Francisco de Paula Santander de Cúcuta, $\mathrm{N}^{\circ}$ de contrato: 012-2018. El texto muestra los resultados de uno de los trabajos realizados a través de la tesis de maestría en Práctica Pedagógica, articulado a este macro-proyecto.

*** Universidad Francisco de Paula Santander de Cúcuta. Cúcuta, Colombia. E-mail: jesusurbina@ufps.edu.co

(1) orcid.org/0000-0002-5262-9527 Google Scholar

**** Universidad Francisco de Paula Santander de Cúcuta. Cúcuta, Colombia. E-mail: lucyraimabc@hotmail.com

(1) orcid.org/0000-0002-9755-3650 Google Scholar
}

rev. eleuthera. Vol 22 No. 1, enero-junio 2020, 47-66 
La violencia escolar desde las representaciones sociales de estudiantes de la institución educativa colegio nuestra señora de Belén

\section{Introducción}

La violencia es un tema que ha ocupado una amplia cobertura en los medios de comunicación y, en este sentido, ha sido objeto de discusión en distintos campos disciplinares, especialmente en el ámbito académico. En particular, este texto aborda la violencia que se teje en la cotidianidad de la escuela, desde las representaciones que le otorgan sus protagonistas: un grupo de niños y niñas de una institución educativa ubicada en un sector marginal de la ciudad de Cúcuta, capital del departamento de Norte de Santander, ubicada en la frontera colombo-venezolana.

La naturaleza del fenómeno de la violencia se ha explicado a través de factores sociales, culturales, históricos, políticos y psicológicos. Sin embargo, preocupa la tendencia común a trivializar el tema y convertirlo en parte de la cotidianidad, afectando el adecuado desarrollo de la vida humana, especialmente dentro de la escuela. Persiste una normalización de los actos violentos, de diversas formas de exclusión, intimidación, abuso o amenaza entre iguales dentro del contexto escolar.

Teniendo en cuenta que la violencia surge y acontece en diversos ámbitos y sectores de la sociedad, el incremento de casos de violencia en el ambiente escolar hace que ésta deje de ser un espacio de seguridad para convertirse en un espacio de vulneración, silencio y omisión. De allí que Ortega, Mora y Mora-Merchán (1995) consideren que la conducta agresiva de los niños y jóvenes está condicionada por la estructura escolar y sus métodos pedagógicos, así como por todo un conjunto de factores políticos, económicos y sociales que lo afectan.

Diversos trabajos persisten en estudios descriptivos de corte cuantitativo que por lo general reseñan estadísticas del maltrato entre pares o formas de violencia contra niños y niñas y, en muchos casos, de los docentes contra sus estudiantes. Aunque muestran cifras alarmantes sobre el fenómeno, no profundizan en las causales de orden social, emocional y relacional que gestan este tipo de comportamientos. Se hace necesario indagar en el asunto desde otras plataformas epistemológicas, de modo que favorezcan la comprensión del fenómeno desde los mismos sujetos, inmersos en estas realidades hostiles. Estudiar los fenómenos sociales desde la teoría de las representaciones sociales ofrece la posibilidad de entender estos tópicos en la realidad en que viven los sujetos de esta representación (Jodelet, 1986; Mireles, 2011).

Ahora bien, las interacciones y las relaciones interpersonales pueden comprenderse dimensionando las condiciones sociales e institucionales en que se producen. En este sentido, la escuela podría proporcionar estrategias básicas a los estudiantes para facilitar su convivencia, fomentar sus procesos de socialización y consecuentes competencias ciudadanas. Sin embargo, suele ocurrir que a la hora de recorrer este camino y alcanzar ciertas metas se presenten altibajos, incluyendo la violencia en sus múltiples formas. 
Este problema está afectando la vida cotidiana de la comunidad académica dificultando las buenas relaciones, el desempeño de los estudiantes y la calidad de la enseñanza. De este modo, es preciso contemplar las representaciones sociales que elaboran los estudiantes acerca de la violencia escolar para hacer más comprensiva la problemática y así tomar acciones que hagan más partícipes a los estudiantes, como puede ser la adopción de un enfoque integral que involucre a los padres, los educadores y la comunidad.

Dentro de lo estipulado en el informe sobre violencia escolar en América Latina y el Caribe para UNICEF y Plan Internacional (Eljach, 2011), se especifica que la violencia ejercida contra los niños, niñas y adolescentes (NNA) es uno de los problemas más graves presentes en los contextos familiares, institutos para la protección, centros de privación de libertad, calles y, fundamentalmente, las escuelas.

El castigo corporal es una práctica que se ha naturalizado con el pasar de los años como un método de disciplina y aprendizaje; frente a este fenómeno, la violencia psicológica o maltrato emocional aparece como una tendencia en constante aumento y difícil de controlar, ya que se trata de prácticas poco visibles pero que atentan contra la autoestima de los individuos promoviendo patrones violentos de relación. En consecuencia, las prácticas violentas en las escuelas configuran una compleja red de agresiones que evidencian la crisis relacional entre los estamentos de la comunidad educativa.

En el presente texto, esta problemática se estudia en el entorno escolar del Colegio Nuestra Señora de Belén a partir del análisis del “Observador del estudiante”, documento de carácter institucional que se utiliza para registrar aquellos hechos académicos o disciplinarios de los estudiantes considerados como "faltas en el Manual de Convivencia”. Es importante señalar que la Institución no cuenta con otra fuente o instrumento que facilite información acerca del comportamiento de los alumnos.

Hasta 2014, la Institución Educativa contaba con aproximadamente 576 estudiantes correspondientes a la jornada de la mañana, de los cuales el comité disciplinario sancionó a 30, estipulando una matrícula condicional y compromisos individuales por motivo de conductas violentas reiteradas contra los diferentes actores educativos (estudiantes, docentes y administrativos).

De todos los estudiantes amonestados, el $57 \%$ pertenecían a $8^{\circ}$ grado; siendo 8 -C el curso que presentaba el mayor número de sancionados. Por su parte, el $27 \%$ de los amonestados eran de $9^{\circ}$ grado, el $13 \%$ de $10^{\circ}$ grado y el $3 \%$ de $11^{\circ}$ grado. La edad de los estudiantes con repetitivas conductas agresivas promedió los 13 años de edad. A nivel de género, el $73 \%$ de los estudiantes sancionados pertenecían al género masculino mientras que el $27 \%$ correspondía al género femenino. 
La violencia escolar desde las representaciones sociales de estudiantes de la institución educativa colegio nuestra señora de Belén

De este modo, el "observador del estudiante" identifica que uno de cada 20 estudiantes ha recibido rigurosas sanciones por parte de los docentes y directivos de la institución debido a su actuar violento: agresión física y verbal a estudiantes y a docentes porte de armas corto punzante, hurto y daño a la propiedad privada.

En este contexto problemático surge la pregunta nuclear de este texto: ¿Cómo representan la violencia escolar un grupo de niños y niñas de una escuela pública en un barrio popular de Cúcuta?

\section{Horizonte teórico y conceptual}

\section{Violencia escolar}

La violencia en el contexto escolar es un fenómeno que ha sido susceptible de diversos debates y producción intelectual en trabajos investigativos de ciencias como la psicología, la sociología, la educación e incluso la medicina, desde las que se ha abordado el problema tomando como base diferentes constructos teórico-metodológicos. Asimismo, para el abordaje de la violencia escolar es importante adquirir cierta comprensión sobre el ámbito educativo, es decir, poder aceptar y reconocer que una cosa es la problemática de la violencia escolar en tanto que fenómeno a estudiar y otra las representaciones sociales acerca de la violencia escolar.

Los siguientes aspectos pueden considerarse como descripciones relacionadas con la problemática en cuestión:

(...) la violencia interpersonal entre escolares puede manifestarse de forma verbal, física, sexual, psicológica, relacional y sobre las propiedades de los otros. Es verbal cuando se manifiesta mediante insultos y palabras de desprecio; física, cuando se expresa a través de golpes; sexual, al afectar comportamientos, actitudes y percepciones eróticas sexuales (Ortega, 2002); psicológica, al presentarse a través de ataques a la identidad de la persona, bien en forma de amenazas o de chantajes; relacional, al darse a través del aislamiento, el rechazo y la indiferencia; finalmente, hablamos de robos cuando la violencia supone la sustracción de las propiedades de los otros. (Ortega, 2005, p. 789)

De igual manera, los trabajos descriptivos han permitido cuestionar algunos factores que se encuentran asociados al fenómeno, por ejemplo la edad y el género de los implicados; algunos de los estudios sostienen que la violencia escolar por lo general afecta más al género masculino, no en razón de que el género femenino no sea objeto de violencia, sino porque la incidencia disminuye conforme van creciendo en edad (Ortega, 2005). 
Los autores sostienen que ha predominado la tendencia victimizatoria que simplemente ha estereotipado el fenómeno y que de alguna manera el término bullying ha servido para conceptualizar la violencia. En este sentido, el bullying ha sido el concepto que ha tendido a configurar la victimización escolar, así como su objeto de estudio (Flores y Retamal, 2011).

Entre las principales consecuencias para comprender la problemática, se resalta el término denominado "estructura victimizatoria" que se entiende como la deformación del concepto de violencia escolar por la noción abstracta de bullying que simplifica el fenómeno:

\begin{abstract}
El modelo Smith, el School Bullying, corresponde a un acercamiento psicosocial de la violencia escolar focalizado en la 'agresión' y el 'hostigamiento' entre los estudiantes. Recuérdese que el término bullying en inglés es utilizado en primer lugar en la definición de relaciones de hostigamiento en el mundo laboral como factor de estrés exclusivamente referido a la autodepreciación emocional de la víctima. En la escuela, el psicólogo Dan Olweus denuncia una forma específica de agresividad entre estudiantes, el school bullying, y lo define principalmente desde el estudiante víctima de hostigamiento, que habría de sufrir una exposición continua y a largo plazo de actos negativos por parte de otro u otros estudiantes. (Flores y Retamal, 2011, p. 323)
\end{abstract}

Dilucidar este punto de vista es una acción reflexiva que se asemeja a las representaciones sociales, al evidenciar que el término bullying ha sido estereotipado y victimizado en diferentes ámbitos; por esta razón, se hace necesario usar el concepto de violencia escolar.

La noción de bullying "no representa todo el fenómeno de la violencia escolar, pero tampoco podríamos decir que es solo una parte y menospreciar su riqueza imaginaria impregnada en las percepciones subjetivas de los actores" (Flores y Retamal, 2011, p. 323). Desde esta visión, el clima escolar se visualiza como un contexto propicio para desarrollar el estudio sobre la violencia escolar interdisciplinariamente, pues las percepciones que alumnos y profesores poseen acerca de este fenómeno determina no solo el aprendizaje en las aulas sino fundamentalmente sus relaciones (López, Bilbao y Rodríguez, 2011)”.

De igual manera, Galtung (2004) señala que la violencia puede ser abordada considerando sus tres formas de expresión: violencia directa, por ser un fenómeno con manifestaciones visibles de agresión tanto verbal como física; violencia cultural por referirse a aquellos actos que son promovidos, legitimados y justificados por los diferentes actores de la comunidad educativa y, por último, la violencia estructural, al tratarse de comportamientos impuestos y reforzados por las estructuras sociales que limitan las necesidades de supervivencia, la libertad, el bienestar y la identidad. 
La violencia escolar desde las representaciones sociales de estudiantes de la institución educativa colegio nuestra señora de Belén

Desde la postura de Baggini (2011), la violencia escolar la constituyen todas aquellas relaciones interpersonales conflictivas que se dan en el contexto educativo (teniendo en cuenta su entorno social próximo), desde donde emergen lazos de dominación basados en una lógica que responde a la agresividad entre actores de la comunidad académica, provocando situaciones victimizantes caracterizadas en la mayoría de los casos por el daño físico, psicológico y emocional que afectan a la víctima y al victimario.

También es importante incluir la definición de bullying o acoso escolar propuesta por el Ministerio de Educación Nacional en la Ley 1620 de 2013:

\begin{abstract}
Conducta negativa, intencional metódica y sistemática de agresión, intimidación, humillación, ridiculización, difamación, coacción, aislamiento deliberado, amenaza o incitación a la violencia o cualquier forma de maltrato psicológico, verbal, físico o por medios electrónicos contra un niño, niña o adolescente, por parte de un estudiante o varios de sus pares con quienes mantiene una relación de poder asimétrica, que se presenta de forma reiterada o a lo largo de un tiempo determinado. (p. 1)
\end{abstract}

De acuerdo con Potocnjak, Tomicic y Berger (2011), en una situación violenta es posible que los roles de los involucrados sean intercambiables y busquen diferentes fines: a) víctimas: sufren directamente la intimidación; b) intimidadores: son los que ejecutan y lideran la intimidación; c) reforzadores: refuerzan la intimidación con risas, burlas, incitaciones y sirviendo de audiencia; d) defensores: frenan la intimidación interviniendo y defendiendo con medios no agresivos a la víctima o la consuelan cuando la agresión termina; f) externos: cuando se presentan las agresiones no están presentes o simplemente se alejan de las mismas.

Los estudios también han señalado que la agresividad es vista como un problema de adaptación, que corresponde a una práctica aprendida en contextos donde las conflictividades están inmersas y latentes, existen dificultades en la comunicación, escaso afecto y apoyo por parte de los padres; así como naturalización de la violencia como forma cotidiana de relación que provoca una deficiente regulación de la conducta de los hijos.

Otros estudios sobre violencia escolar permiten profundizar en los anteriores planteamientos. Orellana y García (1996) abordan el tema de las representaciones sociales de la violencia escolar en el Perú; Álvarez, Cárdenas, Frías y Villamizar (2007) profundizan en el tema de las actitudes hacia la violencia en estudiantes de la ciudad de Bucaramanga; Góngora y Pérez (2007) el acoso escolar; Chaux (2003) la agresión reactiva; De Zubiría (2009) analiza la violencia en los colegios de Bogotá y Hoyos, Aparicio, Heilbron y Schamun (2004) las representaciones sobre el maltrato. 


\section{Representaciones sociales}

Las representaciones sociales son un conjunto de ideas y conocimientos por medio de los cuales las personas comprenden, interpretan y actúan en la realidad (Moscovici, 1979). Estos conocimientos son parte del pensamiento de sentido común y se tejen con las ideas que la gente organiza, estructura y legitima en su vida cotidiana. Así, una representación tiene el carácter de social por dos razones: se generan en grupo y orientan las acciones de las personas. El contexto con el que los jóvenes interactúan influye en la forma como ellos construyen la representación social de su realidad, es decir, todo aquel conocimiento que se va almacenando en la historia y que va determinando las diferentes acciones en las familias, instituciones educativas y comunidades. Lo anterior corresponde con los planteamientos de Moscovici (1979), sobre como la representación es una forma de conocimiento y destaca la importancia de su función social, de los procesos comunicativos y de la interacción social: toda representación está compuesta de figuras y expresiones socializadas.

Del mismo modo, una representación social es una organización de imágenes y de lenguaje porque recorta y simboliza actos y situaciones que son o se convierten en comunes, desde la interacción social. Por tanto, Moscovici (1979) afirma que la génesis de una representación social está determinada por la presencia de un evento, fenómeno o situación novedosa, que afecta a un grupo social. Asimismo, Araya (2002) define de esta manera el enfoque clásico o procesual de la representación social como un paradigma que permite la aproximación comprensiva al objeto de representación.

Según Moscovici y Hewstone (1988) las representaciones sociales se definen como:

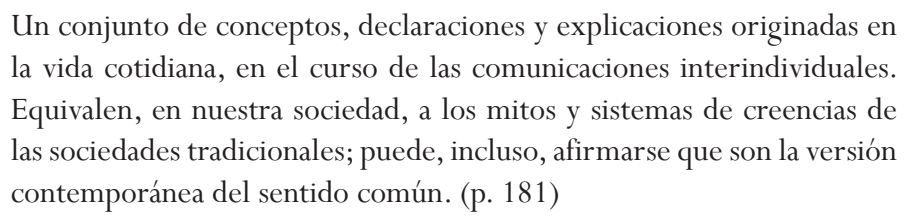

De igual manera, Moscovici (1979) ha señalado que "las representaciones se articulan en torno a tres ejes o dimensiones: información, campo de representación y actitud” (p. 45). De este modo, la información se refiere a la suma de conocimientos poseídos a propósito de un objeto social, así como a su calidad. 
La violencia escolar desde las representaciones sociales de estudiantes de la institución educativa colegio nuestra señora de Belén

\section{Diseño metodológico}

\section{Método}

La presente investigación es de tipo cualitativa, utiliza el enfoque procesual de las representaciones sociales, teniendo en cuenta que privilegia la comprensión social de la cultura y de las interacciones sociales (Araya, 2002), en este caso particular, aborda las representaciones de violencia en la escuela desde la perspectiva de un grupo de niños y niñas matriculados en la Institución Educativa Nuestra Señora de Belén de Cúcuta. En este marco general se tiene en cuenta el carácter comprensivo del fenómeno estudiado "[...] lo que constituye la naturaleza de la experiencia, describirlo e interpretarlo, mediando diferentes significados aportados por cada participante y desde la perspectiva construida colectivamente” (Hernández, Fernández y Baptista, 2014, p. 494).

\section{Universo y participantes}

El Colegio Nuestra Señora de Belén es la institución educativa pública más representativa de la comuna número 9 de Cúcuta (Norte de Santander), uno de los sectores más populares y tradicionales de la capital fronteriza. La población del proyecto es de 554 estudiantes, los cuales pertenecen a la jornada de la mañana. Según el Proyecto Educativo Institucional (2012), el colegio desarrolla sus funciones en un contexto caracterizado por la marginalidad, el desempleo, restricciones en el acceso a la educación, descomposición familiar, conflictos intrafamiliares y desplazamiento forzoso. Los sujetos participantes fueron 20 estudiantes, de los cuales 10 no presentaban reportes disciplinarios y los 10 restantes sí aparecían en los reportes disciplinarios por conductas agresivas contempladas en "el observador del estudiante"; se tomó esta nuestra intencional con el fin de poder contrastar las representaciones de los participantes reportados y no reportados en el "Observador disciplinario", a través de la comparación constante, técnica empleada como herramienta de codificación y análisis de datos por la teoría fundamentada. Ahora bien, el archivo del observador facilitó la selección de los participantes sin criterio estadístico de generalización, sino como muestra intencionada de acuerdo con los siguientes criterios:

$\S \quad$ Que fuesen estudiantes matriculados en el colegio

$\S$ Que 10 de estos niños presentasen anotaciones en el observador del estudiante

$\S$ Que 10 de estos niños no presentasen anotaciones en el observador del estudiante

$\S$ Que mostrasen interés en participar en la investigación. 


\section{Técnicas e instrumentos}

Para el cumplimiento de los propósitos investigativos se empleó como técnica de recolección de la información la entrevista semiestructurada, asimismo, se realizó un grupo de enfoque con única sesión tanto para los estudiantes con comportamientos agresivos como para los que no presentaban reportes por dichas conductas. Finalmente, se realizó una sesión con los 20 estudiantes con el fin de facilitar la interacción entre los participantes y analizar la construcción grupal de los significados.

Por último, se implementó la técnica de las redes de asociación que busca por medio de unas palabras estímulos en la estructura de un mapa semántico, representaciones o eventos donde emergen relaciones que son susceptibles al cambio y que en conjunto producen significados (Figueroa como se citó en Vera, Pimentel y Batista, 2005).

\section{Análisis y discusión de resultados}

Para categorizar y analizar la información se empleó como herramienta la teoría fundamentada, tomando como referente su enfoque cualitativo, teniendo en cuenta que "permite determinar cuál es el núcleo central de las representaciones sociales y sobre qué se estructuran las mismas” (Campo y Labarca, 2009, p. 42). Desde la perspectiva de los participantes se realizó una revisión y comparación teórica del fenómeno como herramienta analítica indispensable para identificar las propiedades y dimensiones de las categorías asociadas a ella. De esto se trata la codificación abierta, un proceso mediante el cual se identifican los conceptos y se descubren en los datos sus propiedades y dimensiones por medio de la descomposición en partes discretas en busca de similitudes y diferencias. Esto permite elaborar conceptualizaciones más abstractas y etiquetar los fenómenos (Strauss y Corbin, 2002).

Posteriormente, a través de la revisión documental se identificaron las siguientes categorías para abordar tanto a estudiantes agresivos como no agresivos: violencia cultural, violencia estructural, clima escolar, violencia entre iguales, violencia directa y prevención de la violencia escolar; así mismo, las respectivas aproximaciones al fenómeno que se han desarrollado desde la teoría de las representaciones sociales en el contexto escolar, sirvieron de hoja de ruta al presente estudio para el respectivo análisis. El análisis de los datos cualitativos se realizó inicialmente de forma manual y luego se utilizó el software ATLAS.ti para segmentar datos en unidades de significado, relacionar conceptos, categorías y temas.

\section{La violencia como forma de supervivencia escolar}

Considerando el concepto de violencia constituido desde la Organización Panamericana de la Salud (2002); es posible deducir que las representaciones expresan algunas dimensiones 
La violencia escolar desde las representaciones sociales de estudiantes de la institución educativa colegio nuestra señora de Belén

sociales y emocionales que transcienden de la idea física de la violencia. Es decir, la violencia desde las representaciones sociales de los estudiantes se refiere al abuso del poder y dominio que se ejerce por medio del maltrato físico y verbal con el objetivo de generar daños psicológicos e inseguridad en la contraparte, la cual moldea a la vez una postura defensiva que manifiesta sentimientos de venganza y rencor contra el victimario.

Es comprensible desde la praxis que la violencia incorpora dentro de sus manifestaciones al maltrato y todos sus matices en función de la intensidad de la situación conflictiva y medios de abordaje; frente a este contexto, el ser humano busca la adaptación a estos entornos difíciles bajo sus propias creencias y normas inculcadas, lo cual se vio reflejado en los participantes de la investigación al enfrentarse a los escenarios y ambientes violentos en los cuales se encontraban inmersos. Lo dicho se fundamenta en lo que señala la Organización Panamericana de la Salud (2002), al afirmar que la aceptación de la violencia la constituye el continuo reconocimiento y evolución que las diferentes culturas hacen de su sistema de valores y normas de comportamiento social.

Para los alumnos un abuso escolar es caracterizado por zancadillas, empujones, golpes sin razón aparente, como lo refleja la opinión dada por ellos: "Para mí es pelear, por ejemplo, si les cae uno mal, hablan con el parche para que lo puñaleen" (E1, comunicación personal, 26 de mayo de 2015).

La perpetuación de la violencia tanto grupal como individual no siempre corresponde a un enfrentamiento entre dos personas, en ciertos grupos sociales se ve la violencia como una forma de adaptación, de sobresalir o de infundir respeto entre los miembros de su grupo, siendo que la persona escogida o víctima sea débil ante ellos o se crea débil; es por eso que la violencia también representa ejercicio de poder sobre el otro y en este caso los estudiantes entrevistados con conductas agresivas reconocen que una de las causas es la búsqueda de reconocimiento: "Pues el que busca problemas siempre quiere ganar, entonces para ganar el respeto, para que lo conozcan en el colegio y también para que le tengan miedo en el colegio” (E2, comunicación personal, 22 de mayo de 2015).

Lo anterior también implica que para los estudiantes considerados como agresivos existe plena claridad de sus acciones y de lo que las origina, dependiendo de la forma que elijan para llevarla a cabo, entre lo que destacan según refieren: "Los apodos, que se metan con la familia" golpes "por ejemplo, con puños, patadas, cabezazos" y burlas "se la pasan colocándose apodos" (E3, comunicación personal, 25 de mayo de 2015).

\section{La violencia como medio de aceptación social}

Sumado a lo anterior, referente a la categoría de la violencia cultural que causa la respectiva representación social de la violencia escolar, se describe una asimilación de la conducta violenta 
en los estudiantes como una forma adecuada de demostrar poder ante los demás, en especial a aquellos que consideran débiles, como lo mencionan al expresar que: "A mí me genera alegría, porque están peleando y uno con el parche está alentando: ¡dale, dale, dale!” (E5, comunicación personal, 22, mayo, 2015). De esta manera se evidencia una manera efectiva de generar un precedente y ser reconocido y digno de aceptación dentro de un grupo.

Debido a las presiones laborales y en ocasiones problemas de índole personal, los padres no prestan la atención necesaria a los hijos y, por lo tanto, muchas veces no están al tanto de sus dificultades, comportamientos, gustos, entre otros; tal y como se refleja en esta expresión:

Las familias son groseras, consumen drogas; no les paran bolas, ellos llaman la atención. "Les dedican muy poco tiempo", "les pegan duro, con peroles, sillas, palos; o a veces la mamá o el papá les dicen a los amigos malos que le peguen un susto”. (E6, comunicación personal, 26, mayo, 2015)

Tales atribuciones tienen su asiento en la representación social de la violencia escolar, ya que ésta "es producto y proceso de una actividad mental por la que un individuo o un grupo reconstituye la realidad que enfrenta y le atribuye una significación específica” (Abric, 2001, p. 12).

Es importante también resaltar que el análisis de la información puso en evidencia los contextos familiares de los participantes con conductas agresivas, este contexto es particularizado por la poca atención, fallas en la comunicación, consumo de sustancias psicoactivas, violencia intrafamiliar y conductas delictivas y de castigo severo; dicha información concuerda con lo postulado en Chaux (2012), quien señala que los individuos expuestos a violencia en sus barrios o familias (por ejemplo, observando peleas o escuchando palabras soeces) tienden a desarrollar con más frecuencia manifestaciones y conductas agresivas.

\section{De víctima a victimario}

Por su parte, las representaciones sociales sobre el perfil de las víctimas de violencia escolar construidas por los estudiantes participantes concuerdan con las características establecidas en la teoría planteada por Ortega, Castelán y Ramírez (2006), quienes la describen como una persona retraída, ignorada, excluida, con baja autoestima y poca motivación, lo que a su vez provoca el aislamiento social de la víctima. Tanto para los autores citados como para los participantes, dichas características no son estáticas, ya que pueden generar una conducta reactiva de la víctima, es decir, puede fácilmente convertirse en agresor a raíz de los ataques de los que fue objeto. 
La violencia escolar desde las representaciones sociales de estudiantes de la institución educativa colegio nuestra señora de Belén

\section{"Uno da una opinión, sea buena o mala, y empiezan a insultarlo"}

Comprender las representaciones de la violencia escolar desde las entrevistas realizadas dio pie para acercarse a la categoría clima en el aula. En dicha categoría se resalta el irrespeto al otro, el cual se refiere no solo a insultos verbales o físicos, sino que es algo esencialmente relacionado con el ser víctima de ataque por pensar diferente, por hablar o lucir diferente.

Para atender las susceptibilidades que puede provocar una opinión en un escenario de violencia escolar, es pertinente comprender que "el sujeto y el objeto no son fundamentalmente distintos" (p. 12), dice Moscovici (como se citó en Abric, 2001), esto significa que si un individuo (o un grupo) expresa una opinión (es decir, una respuesta) respecto a un objeto o a una situación, dicha opinión en cierta forma es constitutiva del objeto, lo determina.

El desempeño cotidiano lleva a la adaptación de la violencia escolar, en consecuencia es a lo largo del proceso adaptativo que se crea la representación social de la violencia. De hecho, la representación social "es producto y proceso de una actividad mental por la que un individuo o un grupo reconstituye la realidad que enfrenta y le atribuye una significación específica” (Abric, 2001, p. 12). Esta es la representación social sobre la adaptación a la violencia escolar, un conjunto de atribuciones que giran en torno al agresor y su poder.

\section{Naturalización de la violencia escolar}

Para complementar los hallazgos de la entrevista por medio del grupo focal, se amplió la perspectiva de los estudiantes que han presentado conductas agresivas ante el concepto de violencia escolar; en este punto fue difícil poder definir comportamientos bajo el calificativo de "violentos", puesto que las groserías, insultos apodos, entre otras cosas, constituyen su lenguaje cotidiano, y se hace evidente en la forma como se saludan entre ellos, haciéndolo propio y sirviendo como referente de normalidad o cotidianidad.

Banchs (1986) explica que una vez es representado un objeto este pierde su naturaleza desconocida e incierta para convertirse en algo identificable en la cotidianidad. "Reconstruimos así en nuestro interior lo que se encontraba fuera de él y, al apropiarnos de ese objeto, éste se integra a nuestro marco de referencia familiar perdiendo su carácter amenazador” (p. 33). Esto significa que la grosería, altanería, insultos y apodos se reconfiguran adquiriendo cualidades de aparente normalidad.

\section{Emociones y violencia}

De igual manera, se halló que la intolerancia asociada a lenguajes no verbales entre los estudiantes producen emociones que van desde la rabia por el abuso, destacando que dicha 
activación emocional no solo proviene de las acciones violentas perpetuadas hacia sí mismos, sino también hacia los demás, dependiendo de qué tan amenazantes o insultantes sean sus interpretaciones y como estas mismas guíen la impulsividad y la emocionalidad, arraigada a ello como ejemplo de esto según refieren en lo expresado en el grupo focal: "A mí me genera rabia, pero al saber que llega la policía a hacer requisas, pues nosotros no hemos hecho nada” (E7, comunicación personal, 22 de mayo de 2015).

Para otros, la emoción que predomina es la indignación ante la injusticia de ser condenados o sancionados por las faltas de otros como se evidencia en la opinión citada anteriormente.

Sin embargo, también se evidencian opiniones opuestas en algunos de los participantes de la investigación al considerar que algunas de la medidas propuestas por las instituciones tienen como propósito garantizar la seguridad de toda la comunidad estudiantil.

\begin{abstract}
No siento desconfianza porque no podemos negar que hay alumnos con cuchillos, que hay alumnos con marihuana dentro del colegio, y que los he visto, no lo podemos negar, entonces cuando llegan ellos acá, dicen: "Por favor, contra la pared y abran sus bolsos"; y así se han llevado puñales y marihuana; ellos saben con quien se están metiendo; en el colegio hay problemas graves (...) hablemos con sinceridad. (E8, comunicación personal, 26 de mayo de 2019)
\end{abstract}

Sin embargo, para algunos la emoción más significativa es la indiferencia ante lo que sucede a su alrededor, corroborado por la siguiente expresión: "A mí no me genera nada (...) normal; porque no es con usted sino con las otras personas, o sea no es problema suyo” (E9, comunicación personal, 22 de mayo de 2019).

Dentro de las emociones también se encuentra la alegría, lo que indica que en cierto nivel la violencia es vista como una conducta “aceptable", teniendo en cuenta la necesidad de sobrevivir en un medio de constantes ataques, en donde la identificación y el apoyo a un grupo determinado es lo más importante, sin importar el costo.

Para aceptar y comprender tales situaciones es necesario recordar que las actitudes defensivas de los estudiantes y la expresión notable de sus reacciones emocionales encuentran su asiento en las representaciones sociales en tanto que característica especifica de las mismas, pues las representaciones sociales están sometidas a la lógica cognitiva y la lógica social. Pueden ser definidas como construcciones socio cognitivas, regidas por sus propias reglas. La coexistencia de ambas permite dar cuenta y comprender por ejemplo por qué la representación integra a la vez lo racional y lo irracional; también porque tolera e integra contradicciones aparentes y porque los razonamientos que genera pueden aparecer como "ilógicos" o incoherentes (Abric, 2001). 


\section{La violencia psicológica como medio de intimidación escolar}

Ahora bien, otra de las categorías de análisis que surgió en el presente estudio se refiere a las diferentes formas y niveles en los que se puede ejercer la violencia causando daño en la víctima. Se destaca la violencia psicológica ejercida a través de insultos y ofensas que afectan la autoestima: "Se insultan vulgarmente porque les cae mal o insultan a la mamá" (E10, comunicación personal, 22 de mayo de 2015).

Este tipo de actitudes agresivas también se presentan entre docentes y estudiantes:

Los estudiantes contra estudiantes y contra docentes, pero con los
profesores solo se da la agresión verbal, insultos o expresiones elevadas
de tono de partes de los docentes como "acá se hace lo que se ordena",
"quien orienta o dicta la clase soy yo", "a mí se me respeta". (E11,
comunicación personal, 22 de mayo de 2015)

Ante esta manifestación de violencia en algunas ocasiones las acciones van orientadas a buscar la ayuda de los docentes, pero la mayoría de las opiniones es que no se toman las medidas adecuadas, pues desde sus puntos de vista y experiencia la violencia no se puede evitar o erradicar, ya que cualquier cosa que para el otro no sea correcta o de su agrado puede ser el detonante; lo que es corroborado por afirmaciones como:

Pues no, porque siempre va a haber un detonante que genera violencia. Como dicen en las redes sociales: el que no hizo Bullying en su adolescencia, no fue nadie; entonces siempre va a haber algo porque pelear y más en las comunas. (E12, comunicación personal, 22 de mayo de 2015)

\section{Medios de comunicación como causantes de la violencia escolar}

La influencia de los medios de comunicación siempre ha sido objeto de preocupación de padres y docentes en pleno auge de la novedad de las redes sociales, medio que favorece la vulneración o perpetración si se tiene en cuenta que se considera que: "Como dicen en las redes sociales: el que no hizo bullying en su adolescencia, no fue nadie”. Es decir, el concepto de bullying se hace extensivo o popular alcanzando "su riqueza imaginaria impregnada en las percepciones subjetivas de los actores” (Flores y Retamal, 2011, p. 323); lo que lleva a afirmar que el concepto de bullying se representa de manera extensa provocando e instigando la victimización y, en consecuencia, fortaleciendo la "estructura victimizatoria".

Los padres de familia y maestros suelen coincidir en que la principal fuerza externa que influye en los comportamientos de los estudiantes es la situación actual de violencia a las que 
se ven expuestos los estudiantes, siguiendo en importancia la influencia de la televisión, la computadora y los videojuegos; lo cual es acorde con lo afirmado por Albaladejo (2011).

En el momento en que se hace popular el uso del concepto de bullying a través de las redes sociales, se hace evidente que "los seres humanos son cualquier cosa menos receptores pasivos, piensan autónomamente y producen y comunican constantemente representaciones... (en todas partes) la gente está haciendo observaciones críticas, comentando, confeccionando, “filosofías" no oficiales (...)” (Moscovici como se citó en Banchs, 1986, p. 28), provocando, muy seguramente, deformaciones intencionadas del término.

El problema del aislamiento del estudiante pasivo en relación con el agresor tiene su fundamento en su incursión en áreas donde es más probable el desencadenamiento de situaciones de riesgo. Tales situaciones evidentemente se configuran en lugares poco controlados; a decir de las conductas violentas ocurren con mayor frecuencia, además del salón de clase, en los pasillos y baños (Valadez y González, 2007).

Entonces, una representación hegemónica de la violencia escolar, tomando la idea de Moscovici sobre regiones de realidad que coexisten en una representación, permite plantear la representación sobre la hegemonía de la violencia escolar y como tal su figuración también es espacial, estableciendo con ello regiones de realidad violentas y excluyentes.

Habría que agregar a la discusión que Valadez y González (2007) sostienen que a los estudiantes les cuesta ser víctimas de maltrato, lo que se percibe como vulnerabilidad y es preferible guardar silencio como medida de autoprotección. Esto no hace sino silenciar los indicadores de victimización.

\section{Rol de los actores en la violencia escolar}

Dentro del conflicto de la violencia escolar las actitudes de los actores, es decir, tanto víctimas como agresores, refuerzan aún más su rol o su posición, de acuerdo con lo encontrado, la implicación de los sujetos hay quien se caracteriza por ser testigo activo, si el sujeto ayuda o apoya al agresor es lo que se conoce como el "respaldo del parche"; por lo tanto es responsable indirecto del acto violento cometido.

La actuación del núcleo familiar con respecto a la violencia vivida dentro de la institución indica que la visión del conflicto que tienen los padres es que a un golpe se debe responder con otro golpe igual o de mayor intensidad; tanto así que son los mismos padres los que incitan a su hijos a responder a "no dejarse" tal y como lo comprueba la siguiente afirmación: 
Una vez una pelada me la tenía montada, yo le conté a mi papá, él me dijo: si la estúpida esa se sigue metiendo con usted, péguele, yo pongo la cara por usted y punto; y eso es lo que me ha dicho desde que yo estoy en la escuelita. (E13, comunicación personal, 25 de mayo de 2015)

A lo anterior habría que agregar el desencadenamiento de una situación social de aislamiento y de aguda impopularidad, llegando a encontrarse entre los alumnos con mayor rechazo dentro del grupo, situación que viene dada su selección como víctimas, base de la victimización.

También es posible afirmar que tanto en el lugar de la víctima o el victimario existe un proceso de representación para su condición, emergiendo las representaciones sociales como estrategia adaptativa y así hacer frente a las circunstancias. De este modo, “(...) los seres humanos tratan de aprehender y entender las cosas que los rodean y de resolver los rompecabezas comunes sobre su nacimiento, su cuerpo, sus humillaciones, el cielo que ven, el humor de sus vecinos y el poder al cual se someten” (Moscovici como se citó en Banchs, 1986, p. 29).

\section{Conclusiones}

Las representaciones sociales de los estudiantes agresivos están construidas a partir de la aceptación de la violencia como una realidad cotidiana, además de una herramienta para lograr tener aceptación e imponer respeto en un grupo donde la agresión es respondida con una acción de igual magnitud y que es esencial para demostrar fortaleza y dominio sobre los otros. De igual manera, es vista como una postura que asume un rol de interacción en un contexto que los obliga a ser víctimas o victimarios, el cual es apoyado por una crianza que se efectúa de manera negativa, y en la cual las creencias, prácticas y valores del núcleo familiar, justifican, agreden y refuerzan conductas violentas.

Para los estudiantes con comportamientos no agresivos, las representaciones sociales en torno a la violencia escolar son consideradas como un fenómeno producto de la intolerancia y el irrespeto a las diferencias del otro, centrando las causas en la falta de valores, de comunicación y en el deseo de reconocimiento que, según la percepción de los participantes, la conducta violenta es alentada tanto por el ambiente familiar hostil como por la opinión de los padres, quienes apoyan dichas acciones con tal de que se defiendan de las agresiones de otros, siendo este el ejemplo que más abunda en los hogares; se deduce que la violencia es aprendida de las figuras paternas o de quien hace sus veces.

La diferencia entre las representaciones sociales sobre la violencia escolar de los estudiantes agresivos y los no agresivos, muestra que para los primeros la violencia es una alternativa con la que han aprendido a convivir, ya que se ha convertido en el mecanismo de sobrevivencia en un ambiente en el que las conductas violentas son aceptadas, justificadas y fácilmente manifiestas ante cualquier situación por menos amenazadora que sea; por lo tanto, existe la percepción 
de que las estrategias de prevención o eliminación de las conductas violentas dentro de la Institución Educativa Nuestra Señora de Belén, no han tenido ni tendrán efecto positivo alguno.

Por otro lado, los resultados son consistentes con Valadez y González (2007), quienes sostienen que a los estudiantes les cuesta trabajo hablar cuando han sido víctimas de maltrato, lo que se percibe como vulnerabilidad y es preferible guardar silencio como estrategia de autoprotección, lo que contribuye a silenciar los indicadores de victimización que se pueden registrar en el observador del estudiante.

El silencio se constituye como representación social de la violencia escolar al configurar modos de marginación o relación en torno a la víctima. De este modo, el estudiante percibido como quieto o callado es conducido hacia el aislamiento bajo la óptica y el trato de la realidad socialmente dominante de la violencia escolar.

Autores como Valadez y González (2007) advierten que la conducta maltratadora se presenta de manera cíclica entre los estudiantes, periodos relativos de tiempo en que la víctima goza de periodos de aparente tranquilidad mientras los agresores dirigen sus acciones de intimidación a otro u otro estudiante; a lo que se podría agregar que en dichos periodos se establece el silencio de la víctima y la marginalidad de la misma.

La profundidad del conflicto estaría a la par con la profundidad con que se acoge la representación social de la violencia, en este sentido, la naturaleza social de una representación viene dada por la relación sujeto-objeto que determina al objeto mismo, pues una representación siempre es la representación de algo para alguien (Abric, 2001).

Representar la escuela como un escenario para resolver conflictos es un paso necesario en la construcción de ciudadanía (Arango, 2008); no extraña que el fortalecimiento de estos valores ciudadanos sea mayor cuando se integra a la actividad escolar a las familias, estudiantes y docentes en actividades más participativas en torno a la problemática de la violencia escolar.

\section{Referencias}

Abric, J. (2001). Prácticas sociales y representaciones. Ciudad de México, México: Coyoacán.

Albaladejo, N. (2011). Evaluación de la violencia escolar en educación infantil y primaria (tesis doctoral). Universidad de Alicante, Alicante, España.

Álvarez, L., Cárdenas, A., Frías, P. y Villamizar, M. (2007). Actitudes hacia la violencia social entre iguales y su relación con variables socio-demográficas en tres grupos de estudiantes de secundaria de la ciudad de Bucaramanga. Revista Colombiana de Psicología, 16, 127-137. 
La violencia escolar desde las representaciones sociales de estudiantes de la institución educativa colegio nuestra señora de Belén

Arango, L. (2008). Representaciones y prácticas sobre ciudadanía en estudiantes de octavo grado de educación básica secundaria de tres planteles educativos pertenecientes a estratos sociales diferentes (trabajo de pregrado). Universidad de Antioquia, Medellín, Colombia.

Araya, S. (2002). Las representaciones sociales. Ejes teóricos para su discusión. San José, Costa Rica: FLACSO-ASDI.

Baggini, I. (2011). ¿Qué significados tiene la violencia en la escuela?: estudio sobre las representaciones sociales de la violencia escolar en una escuela secundaria de la ciudad de México. Ciudad de México, México: Latinoamericana de Ciencias Sociales Sede Académica.

Banchs, M. (1986). Concepto de representaciones sociales. Análisis comparativo. Revista Costarricense de Psicología, 8(9), 90-105.

Campo, M. y Labarca, C. (2009). La teoría fundamentada en el estudio empírico de las representaciones sociales: un caso sobre el rol orientador del docente. Revista Opción, 25 (60), 41-60.

Chaux, E. (2003). Agresión reactiva, agresión instrumental y el ciclo de la violencia. Revista de Estudios Sociales, 15, 47-58. Recuperado de http://redalyc.uaemex.mx/ pdf/815/81501504.pdf

Chaux, E. (2012). Educación, convivencia y agresión escolar. Bogotá, Colombia: Uniandes.

De Zubiría, J. (2009). La violencia en los colegios de Bogotá. Recuperado de http://www. institutomerani.edu.co/publicaciones/articulos/2009/La_violencia_en_los_colegios_ de_Bogot $\%$ C3\%A1.pdf.

Eljach, S. (2011). Informe sobre violencia escolar en América Latina y el Caribe: superficie y fondo. Ciudad de Panamá, Panamá: Plan Internacional y UNICEF.

Flores, L. y Retamal, J. (2011). Clima escolar y gestión compleja del conocimiento: desafíos para la investigación educativa y la política pública en violencia escolar. Revista Internacional de Investigación en Educación, 4(8), 319-338.

Galtung, J. (2004). Violencia, guerra y su impacto. Sobre los efectos visibles e invisibles de la violencia. Recuperado de http: / / www.lopezaso.com/adm/contenido/48.pdf.

Góngora, M. y Pérez, B. (2007). Acosadores escolares o Bullyies en Bogotá: perfil de un comportamiento invisible en ámbitos escolares. Revista Forensis, 292- 306.

Hernández, R., Fernández C. y Baptista, P. (2014). Metodología de la investigación. Ciudad de México, México: McGraw Hill.

Hoyos, O., Aparicio, J., Heilbron, K. y Schamun, V. (2004). Representaciones sobre el maltrato entre iguales en niñas y niños escolarizados de 9, 11 y 13 años de nivel socioeconómico alto y bajo de la ciudad de Barranquilla (Colombia). Psicología desde el Caribe, (14), 150-172. 
Jodelet, D. (1986). La representación social: fenómenos, concepto y teoría. In S. Moscovici. (Ed.), Psicología social II. Pensamiento y vida social (pp. 469-493). Barcelona, España: Ediciones Paidós Ibérica.

Ley 1620 de 2013. (20 de marzo), Por la cual se crea el Sistema Nacional de Convivencia Escolar y Formación para el Ejercicio de los Derechos Humanos, la Educación para la Sexualidad y la Prevención y Mitigación de la Violencia. Diario Oficial N. 48.733.

López, V., Bilbao, M. y Rodríguez, J. (2011). La sala de clases sí importa: incidencia del clima de aula sobre la percepción de intimidación y victimización entre escolares. Revista Javeriana, 11 (1), 91-101.

Mireles, O. (2011). Representaciones sociales. Debates y atributos para el estudio de la educación. Revista Electrónica Sinéctica, 36, 1-11.

Moscovici, S. (1979). El psicoanálisis, su imagen y su público. Buenos Aires, Argentina: Huemul.

Moscovici, S. y Hewstone M. (1986). De la ciencia al sentido común. En S. Moscovici. (Ed), Psicología social II, Pensamiento y vida social. Psicología social y problemas sociales (pp. 679-710). Barcelona, España: Paidós.

Orellana, M. y García, A. L. (1996). Violencia y representaciones sociales en escolares. Revista Peruana de Psicología, 1(1), 26-39.

Organización Panamericana de la Salud. (2002). Informe mundial sobre la violencia y la salud. Revista Asamblea Mundial de la Salud, 6 (12), 255-411.

Ortega, R. (2005).Violencia escolar en Nicaragua: un estudio descriptivo en escuelas primarias. Revista Mexicana de Investigación Educativa, 10(26), 787-804. Recuperado de http: / /www. redalyc.org/pdf/140/14002609.pdf.

Ortega, S., Castelán, A. y Ramírez, M. (2005). Estrategias para prevenir y atender el maltrato, la violencia y las adicciones en las escuelas públicas de la Ciudad de México. Recuperado de http: / / www.rieoei.org/rie38a08.pdf.

Ortega, R., Mora, J. y Mora-Merchán, J. A. (1995). Cuestionario sobre intimidación y maltrato entre iguales. Proyecto Sevilla Anti-violencia escolar. Sevilla, España: Universidad de Sevilla.

Potocnjak, M., Tomicic, T. y Berger, C. (2011). Una aproximación relacional a la Violencia Escolar entre pares en adolescentes chilenos: perspectiva adolescente de los factores intervinientes. Revista Scielo, 20 (2), 39-52.

Strauss, A. y Corbin, J. (2002). Bases de la investigación cualitativa: técnicas y procedimientos para desarrollar la teoría fundamentada. Medellín, Colombia: Universidad de Antioquia.

Valadez, I. y González, N. (2007). Violencia escolar: maltrato entre iguales en dos niveles educativos. Recuperado de http:/ /www.medigraphic.com/pdfs/invsal/isg-2007/isg073g.pdf. 
La violencia escolar desde las representaciones sociales de estudiantes de la institución educativa colegio nuestra señora de Belén

Vera, J., Pimentel, C. y Batista, F. (2005). Redes semánticas: aspectos teóricos, técnicos, metodológicos y analíticos. Revista Universidad Autónoma Indígena de México, 1 (3), 439-451. 